\title{
Orientaciones para el manejo de las especies forestales amenazadas Chanul y Níspero basado en su estado de conservación en el Consejo Comunitario Mayor de Istmina, Chocó, Colombia
}

\section{Guidelines for the management of endangered forest species Chanul and Níspero based in their conservation status in the Consejo Comunitario Mayor de Istmina, Chocó, Colombia}

\author{
William Klinger Brahan, Ing Forst, MSc* \\ RESUMEN
}

El desarrollo de actividades productivas en el municipio de Istmina ha generado una reducción de importantes áreas con cobertura de bosques, afectando la existencia de algunas especies forestales de especial importancia ecológica, dadas sus condiciones de vulnerabilidad y amenaza; tal es el caso del Níspero (Manilkara bidentata) y del Chanul (Sacoglotis procera). A pesar de tenerse información generalizada, el estado actual de existencia de estas especies no se conoce con claridad, por lo que resulta dificil tomar decisiones en materia de su manejo por parte de la autoridad ambiental departamental. Para avanzar en la toma de decisiones acordes con la realidad, la Corporación Autónoma para el Desarrollo Sostenible del Chocó (CODECHOCO) y el Instituto de Investigaciones Ambientales del Pacífico (IIAP), decidieron realizar una investigación, que a partir del conocimiento de las existencias de las especies, posibilitara decisiones acerca de su manejo. Durante la fase de campo se utilizó un muestreo diagnóstico y se establecieron 205 parcelas en dos comunidades del municipio de Istmina, Puerto Salazar y Santa Mónica, dentro de la jurisdicción del Consejo Comunitario Mayor de Istmina (COCOMINSA). Se encontraron 179 individuos de las dos especies, se georreferenció cada individuo, se determinó su estado de desarrollo y su estructura diamétrica. Con fundamento en los resultados se proponen algunas decisiones urgentes como la veda inmediata de largo plazo con la implementación de un programa de preservación para el Chanul y veda inmediata de mediano plazo con un programa de conservación para el Níspero.

Palabras clave: Especies forestales amenazadas; Manilkara bidentata; Sacoglotis procera; Herramientas de manejo; Conservación de bosques; Istmina; Chocó.

\section{ABSTRACT}

The development of productive activities in the municipality of Istmina has generated a significant reduction in forest coverage areas, affecting the stocks of some forest species of special ecological importance, given their vulnerability and threat, as is the case of Nispero (Manilkara bidentata) and Chanul (Sacoglotis procera). Despite widespread information taken, the current state of existence of these species is not known clearly, so it is difficult to make decisions regarding its management by the environmental authority of the department. To move forward in making decisions consistent with reality, the Corporación Autónoma para el Desarrollo Sostenible del Chocó
* Profesor Titular, Universidad Distrital Francisco José de Caldas, Bogotá, y Director General del Instituto de Investigaciones Ambientales del Pacífico (IIAP), Quibdó, Colombia. Recibido: 15 de junio de 2011 Aceptado: 18 de agosto de 2011 


\section{Bioetnia Volumen 8 No 2 (julio-diciembre), 2011}

(CODECHOCO) and Environmental Research Institute Pacific (IIAP) decided to carry out an investigation, which from the knowledge of the stock possible species decisions about its management. During the field phase of sampling was used diagnosis, 205 plots were established two (2) communities in the municipality of Istmina: Puerto Salazar and Santa Monica, within the jurisdiction of the Consejo Comunitario Mayor de Istmina (COCOMINSA). We found 179 individuals of both species, georeference each individual, we determined their stage of development and structure diameter. Based on the results suggests some urgent decisions, immediate closure of long-term implementation of a preservation program for the immediate closure Chanul and medium-term conservation program for the Nispero.

Keywords: Endangered forest species; Manilkara bidentata; Sacoglotis procera; Management tools; Forest conservation; Istmina; Chocó.

\section{INTRODUCCIÓN}

En el municipio de Istmina, Chocó, se han desarrollado históricamente actividades de aprovechamiento minero de relativo bajo impacto sobre el ambiente natural; sin embargo, la reciente introducción de maquinaria pesada en procura de mayores excedentes económicos, ha generado la devastación de una gran cantidad de tierras, trayendo consigo además de la pérdida de cobertura vegetal y con ello los importantes servicios ambientales que presta el bosque, la pérdida de especies forestales catalogadas como amenazadas que son de un alto valor ecológico para Colombia y el mundo.

Lo anterior hace urgente la toma de medidas que promuevan la protección de la base genética del municipio de Istmina, a través de estrategias de manejo de especies de interés especial, lo que no puede hacerse responsablemente sin conocer al detalle el estado de conservación de las mismas. Las especies Níspero (Manilkara bidentada) y Chanul (Humiriastrum procera) por las características de su madera que la hacen resistentes tanto biológica como estructuralmente, han sido sometidas a una fuerte presión antrópica que hoy se traduce en el tamaño de sus poblaciones muy reducido y en su inminente vulnerabilidad según (Cárdenas 2010).

Conscientes de esta situación, el Instituto de Investigaciones Ambientales del Pacífico (IIAP) y la Corporación Autónoma para el Desarrollo Sostenible del Chocó (CODECHOCO), conformaron una alianza para el diseño de estrategias de manejo de estas dos especies, que le permita a la población en general mantener los beneficios que su presencia representa y seguramente tener material vegetal para futuros proyectos de repoblación que decida emprender la autoridad ambiental o cualquier otra entidad para mantener la riqueza forestal de la municipalidad.

\section{INFORMACIÓN GENERAL DE LAS ESPECIES}

\author{
El Chanul
}

Familia: Humiraceae

Nombre científico: Sacoglotis procera Little

Nombres comunes: Chanul, Chano, Chanú, Aceituno, Batea, Nevavac, Diañemiu, Muidotiai, Hispi, Corozo.

Distribución geográfica. Se encuentra desde Costa Rica, Panamá, Guayana, Venezuela, Perú, Ecuador. En Colombia se encuentra en la costa pacífica y en la cuenca de los ríos Calima y Patía.

Aspectos sobresalientes del árbol. Alcanza una altura hasta de $40 \mathrm{~m}$ y un diámetro hasta de $1.20 \mathrm{~m}$. El tronco es recto, cilíndrico con raíces tablares hasta de $2 \mathrm{~m}$ de altura. La corteza externa es de color café rojizo, de textura delgada algo escamosa o en placas con lenticelas. La corteza interna es de color rojizo claro, de sabor amargo y textura fibrosavidriosa. Presenta hojas simples, alternas, elípticas, de borde festoneado con estípulas y pecíolos pequeños. Flores pequeñas y dispuestas en corimbos terminales. Fruto tipo drupa ovoide y comestible. Crece en bosques de colina o elevaciones bajas, en suelos no inundados de la formación vegetal bosque húmedo tropical (bh-T), constituyendo rodales casi puros y a veces asociado con las especies Sande (Brosimun sp) y Cuángare (Virola sp.).

Características externas de la madera. La albura es de color rosado, con transición gradual a duramen de color marrón rojizo con manchas oscuras. Olor característico, ligeramente avinagrado cuando está fresca. Sabor ausente o no distintivo. Brillo de mediano a bajo. Grano recto a entrecruzado. Textura fina a mediana. Veteado suave en bandas longitudinales de color marrón.

Secado. La madera es moderadamente difícil de secar al aire libre presentando rajaduras en los extremos y lados; debido a esto hay que sellar previamente los extremos con sustancias especiales. Se recomienda como horario de secado el programa $\mathrm{S}$ de la Junta del Acuerdo de Cartagena.

Preservación. Es fácil de tratar por cualquiera de los sistemas de inmunización, en los procesos vacíos-presión e inmersión, presenta una retención para albura de $150 \mathrm{~kg} / \mathrm{m}^{3}$ a $200 \mathrm{~kg} / \mathrm{m}^{3}$ y para el duramen de $100 \mathrm{~kg} / \mathrm{m}^{3}$ a $150 \mathrm{~kg} / \mathrm{m}^{3}$ y una penetración parcial periférica.

Trabajabilidad. Es moderadamente difícil de trabajar con máquinas y herramientas comunes, por lo que es recomendable el uso de herramientas con dientes calzados, ya que presenta cristales de sílice, que amellan las herramientas. Tiende con frecuencia a astillarse en las orillas, pero en general ofrece un buen acabado.

Durabilidad. Es una madera moderadamente resistente al ataque de hongos e insectos, con una duración en uso exterior de 5 a 10 años.

Usos actuales. Traviesas para ferrocarril, pisos, cons- 
trucciones pesadas a la intemperie, carrocería, carretería, ebanistería e implementos para agricultura.

Usos potenciales. Para estacones, puentes, construcciones navales, parket, molduras, vigas y soleras.

\section{El Níspero}

Familia: Sapotaceae

Nombre científico: Manilkara bidentata

La balata es una especie botánica nativa de una gran área del norte de Sudamérica, América Central, Caribe. Produce látex de su sabia. Los de Guyana llaman a su madera «corazón púrpura».

Es un árbol grande, alcanzando entre 30 y $45 \mathrm{~m}$ de altura. Las hojas son alternas, elípticas, enteras, de 1-2 dm de longitud, las flores son blancas y se encuentran al comenzar la estación de las lluvias. El fruto es una drupa amarilla, de 3$5 \mathrm{~cm}$ de diámetro, comestible; conteniendo una u ocasionalmente dos semillas. Este árbol exótico cuyo látex blanco es la principal fuente de goma de mascar, se cultiva por su bien conocido fruto comestible.

Usos. Su látex se extrae de la misma manera como se hace con la savia del Hevea brasiliensis árbol de la goma. Luego se seca formando una goma inelástica. Es muy parecida a la gutapercha (producida por un árbol asiático muy emparentado, a veces llamado guta-balatá). A veces se usa para producir la cobertura de pelotas de golf. Es muy bueno pero dura poco en las pelotas de golf, necesitando un nuevo recubrimiento. Se produce mucho en los bancos del río Amazonas y en sus tributarios. Es también muy importante árbol para madera en Puerto Rico. Tiene madera dura, roja, para mueblería y material para la construcción. Es tan densa que no flota en el agua. Para clavar es necesario perforar antes un orificio, por donde va a pasar el clavo. El fruto, parecido al de su pariente Sapodilla (Manilkara zapota), es comestible, con excelente sabor.

\section{METODOLOGÍA}

El desarrollo del proyecto comprendió varias etapas íntimamente ligadas una a otra, se inició con un proceso de concertación, al tiempo que se emprendía una ardua revisión de información secundaria, luego se revisó y elaboró la cartografía base y con fundamento en ella se hizo el diseño de la fase de campo, se levantó la información primaria con un trabajo que vinculó a miembros de la comunidad y finalmente se hizo el procesamiento de la información y la elaboración del documento final.

Concertación. Para tal efecto se canalizaron los esfuerzos a través de los representantes legales de los consejos comunitarios mayores para luego llegar hasta los consejos comunitarios locales. Se realizó una convocatoria amplia de los miembros de consejos y cabildos para llevar a cabo los talleres de concertación, en los que se lograron los acuerdos previstos y se dispuso de los mecanismos para enfrentar la fase de campo del proyecto.

Producción de cartografía básica y temática. Se elaboró un mapa del municipio de Istmina, en el cual se identificaron con puntos las zonas de muestreo; posteriormente se realizó un mapa ampliando esta zona para lograr mayor detalle, pudiendo identificar accidentes naturales. Es precisamente sobre estos últimos mapas en donde se muestra la distribución espacial de las especies forestales identificadas en la etapa de campo, que se georreferenciaron empleando los GPS; además, una vez producidos los diferentes mapas temáticos con la distribución espacial de las especies, a cada mapa se le anexó una matriz, que indica: la especie forestal, las coordenadas geográficas y planas del respectivo árbol al igual que su estado de desarrollo (fustal, latizal o brinzal). Se produjo cartografía para los consejos comunitarios de Suruco Santa Mónica y el consejo comunitario de Puerto Salazar; esta cartografia incluyó la espacialización de las especies forestales amenazadas encontradas en cada una de las zonas.

\section{Fase de campo}

Diseño del muestreo. El personal de apoyo ubicó los sitios de mayor ocurrencia de las especies forestales en estudio; allí se establecieron 205 parcelas de $10 \mathrm{~m}$ x $10 \mathrm{~m}$ distribuidos de manera sistemática a lo largo de los diferentes microambientes (pendientes, planicies y bosques de influencia hídrica), teniendo en cuenta que la distancia mínima entre ellas fuera de $20 \mathrm{~m}$, que no se solaparan, ni existieran en su interior zonas muy alteradas como caminos amplios o claros de bosque. Para la delimitación de las parcelas se utilizó una cuerda. En cada parcela se registraron y midieron todos los individuos de Níspero y Chanul, incluyendo todos los estados de desarrollo (brinzal, latizal y fustal) (Figura 1).

Se hicieron colecciones completas de las dos especies en las parcelas y colecciones adicionales en los sectores aledaños a las parcelas. Se recolectaron cinco ejemplares, preferiblemente en estado reproductivo, que sirvieron de base para asegurar la adecuada identificación de la especie, la información se complementó con registros fotográficos. Toda la información de la especie por localidad (nombre vulgar y científico, usos, descripciones, entre otras), se consignó en una hoja de cálculo de Excel 2007 (Figura 2).

Aspectos estructurales de la población. La densidad se calculó como número de individuos de la especie/área. Para documentar los estados de desarrollo y la morfología de la especie se tuvo en cuenta el diámetro y las estructuras vegetativas y reproductivas (forma, color, longitud y estructura de raíz, tallo, hoja, flores y frutos); lo anterior permitió definir las siguientes categorías: brinzales (individuos con un estado de desarrollo más avanzado que las plántulas); latizales (individuos con un estado de desarrollo avanzado, donde se identifican claramente las características vegetativas parti- 


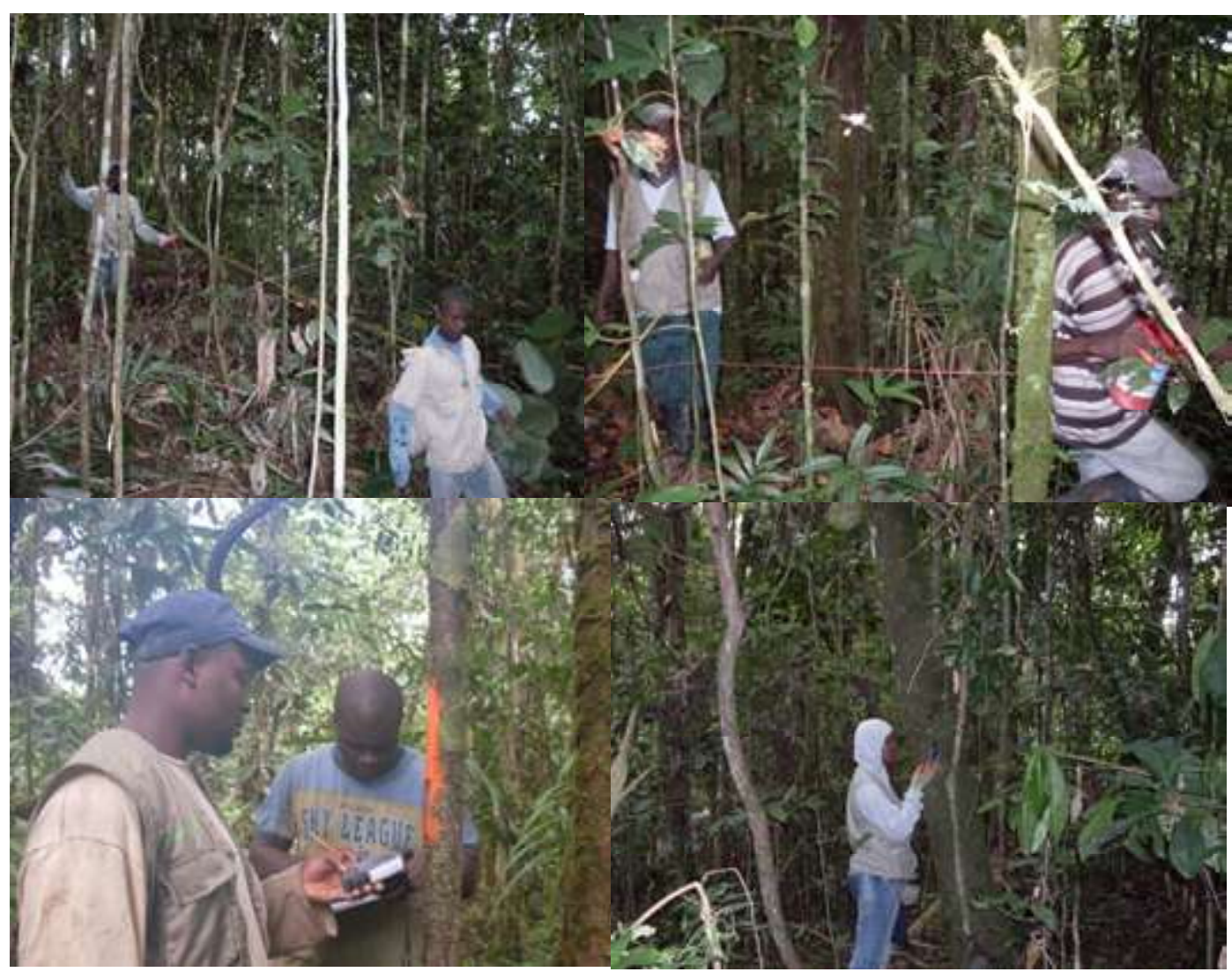

Figura 1. Demarcación de parcelas, georreferenciación y registro de información

culares de la especie, pero sin estructuras reproductivas evidentes o inferidas) y fustales (individuos que presentan alguna evidencia reproductiva como cicatrices, brácteas, frutos o semillas viejas).

Se definieron siete clases diamétricas $(\mathrm{cm})$ de la siguiente manera:

$$
\begin{aligned}
& \text { I: } 0-10 \\
& \text { II: } 10-20 \\
& \text { III: } 20-30 \\
& \text { IV: } 30-40 \\
& \text { V: } 40-50 \\
& \text { VI: } 50-60 \\
& \text { VII: }>60
\end{aligned}
$$

\section{RESULTADOS Y DISCUSIÓN}

A partir de los análisis cartográficos se produjeron los siguientes mapas en los que se espacializan las especies forestales amenazadas que se encontraron en cada una de las zonas, así:

Consejo Comunitario de Suruco Santa Mónica. Espacialización del Chanul en estado fustal 16 árboles; espacialización del Níspero en sus diferentes grados de desarrollo: brinzal 48 árboles, fustal 11 árboles y latizal 14 árboles, para un total de 83 individuos de esta especie forestal.

Consejo Comunitario de Puerto Salazar. Mapa con espacialización del Chanul en sus diferentes grados de desarrollo: brinzal 14 árboles, fustal 11 árboles y latizal 2 árboles, para un total de 17 individuos de esta especie forestal. Espacialización del Níspero en sus diferentes grados de desarrollo: brinzal 43 árboles, fustal 8 árboles y latizal 12 árboles, para un total de 63 individuos de esta especie forestal.

Densidad poblacional. Se tuvieron en cuenta dos sitios de muestreo, uno en el corregimiento de Suruco, vereda Santa Mónica y otro en el corregimiento de Puerto Salazar, vereda de Chigorodó.

Las existencias de individuos de Níspero en la vereda Suruco Santa Mónica sobrepasan los 125 por cada hectárea, lo que para la condición de la especie, representa un alto valor, máxime si estos datos se comparan con los de otras especies forestales amenazadas que en otros estudios realizados en diferentes lugares del departamento han reportado información bastante inferior, como es el caso de las especies: Abarco, Carrá, Choibá y Pino Amarillo en el municipio 


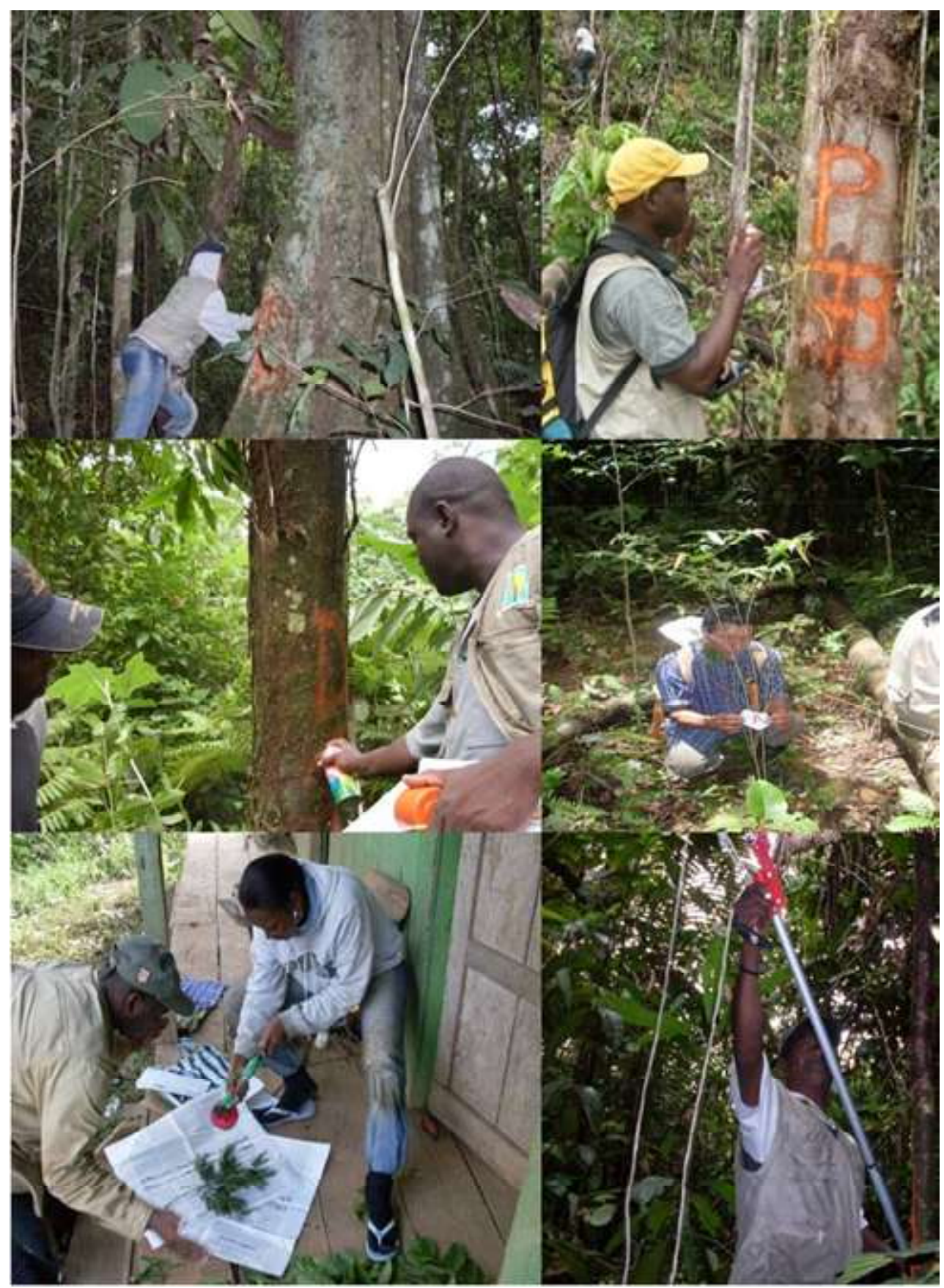

Figura 2. Demarcación de los árboles y colección de muestras botánicas

de Carmen del Darién, otrora emporio forestal del Chocó.

No ocurre lo mismo con las existencias de Chanul, pues algo más de 22 individuos por hectárea, muestra el estado de amenaza en que se encuentra la especie, a pesar de que esta densidad poblacional supera las de la misma especie en otros lugares forestalmente más conservados del departamento del Chocó como es el municipio de Juradó en la frontera con Panamá, donde se registran solo once individuos de Chanul por hectárea. La Tabla 1 detalla la información al respecto de las especies forestales amenazadas que se encontraron en Suruco Santa Mónica.

Las razones que pueden explicar las diferencias encontradas en las existencias de las dos especies, están asociadas a la cultura de uso local de la madera de Chanul en el departamento del Chocó, la especie es ampliamente reconocida y empleada en construcción dada su alta resistencia 
Bioetnia Volumen 8 No 2 (julio-diciembre), 2011

Tabla 1

Existencias por unidad de superficie de las especies forestales amenazadas Níspero y Chanul en Suruco Santa Mónica, Istmina, Chocó

\begin{tabular}{lllcc}
\hline \multicolumn{2}{c}{ Nombre de la especie } & Familia & Número de individuos & $\begin{array}{c}\mathbf{N}^{\circ} \text { de individuos por } \\
\text { hectárea }\end{array}$ \\
\cline { 1 - 2 } común & científico & & 83 & 125.75 \\
\hline Níspero & Manilkara bidentata & Sapotaceae & 15 & 22.73 \\
Chanul & Humiriastrum procera & Humiriaceae & 98 & \\
Total & & &
\end{tabular}

Tabla 2

Especies forestales amenazadas encontradas en Puerto Salazar (Chigorodó), Istmina, Chocó

\begin{tabular}{|c|c|c|c|c|}
\hline \multicolumn{2}{|c|}{ Nombre de la especie } & \multirow[t]{2}{*}{ Familia } & \multirow[t]{2}{*}{ Número de individuos } & \multirow{2}{*}{$\begin{array}{c}\mathbf{N}^{\circ} \text { de individuos por } \\
\text { hectárea }\end{array}$} \\
\hline común & científico & & & \\
\hline Níspero & Manilkara bidentata & Sapotaceae & 63 & 45.32 \\
\hline Chanul & Sacoglotis procera & Humiraceae & 18 & 12.94 \\
\hline Total & & & 81 & \\
\hline
\end{tabular}

mecánica, lo que llevó a la Junta del Acuerdo de Cartagena a catalogarla dentro de los grupos estructurales. A pesar de que la madera del Níspero tiene muy buenas propiedades físicas y mecánicas, su valor de uso es limitado por las dificultades que impone la dureza de la madera a dejarse trabajar por máquinas de ebanistería. Sin embargo, las condiciones tecnológicas de hoy pueden impulsar su uso, tal como ha venido ocurriendo con la madera de Choibá; de allí el peligro en el que se encuentran sus poblaciones.

En la vereda Puerto Salazar también conocida como Chigorodó se encontraron en total 81 individuos de las dos especies amenazadas objeto de estudio. Marcando la misma tendencia del otro sitio de muestreo, existe una mayor cantidad de individuos por hectárea de Níspero que de Chanul, aunque en ambos casos con datos inferiores a los encontrados en Santa Mónica. Los datos hallados durante el trabajo de campo se pueden apreciar en la Tabla 2.

La densidad poblacional del Níspero en la vereda Santa Mónica (125.75 ind/ha) es casi tres veces mayor que en la vereda Puerto Salazar (45.32 ind/ha), lo que evidencia la mayor presión ejercida sobre la especie en un lugar en comparación con el otro. Los datos, de otra parte, ponen en evidencia las mayores ventajas que ofrece Santa Mónica al momento de emprender programas de manejo de la especie tendientes a su conservación.

Para el caso de la especie Chanul, se sigue la misma tendencia al comparar los sitios de muestreo en cuanto a densidad poblacional se refiere, los datos arrojan que este parámetro es casi el doble en la vereda Santa Mónica en comparación con la vereda Puerto Salazar, lo que en términos generales destaca la importancia forestal de Santa Mónica y la necesidad de apoyar proyectos que directa e indirectamente permitan el reconocimiento de su valor desde el punto de vista ecológico.

La Tabla 3 presenta información integrada del territorio colectivo del Consejo Comunitario Mayor de Istmina en el departamento del Chocó (COCOMINSA), los datos corresponden a la suma de lo encontrado durante la fase de campo de la investigación, lo que corresponde al levantamiento de un total de 205 parcelas de $10 \mathrm{~m}$ x $10 \mathrm{~m}$, lo cual arrojo un total de $20.500 \mathrm{~m}^{2}$, equivalentes a 2.05 hectáreas inventariadas entre las veredas de Suruco Santa Mónica y Puerto Salazar.

La información del Consejo Comunitario Mayor de Istmina posibilita la mirada integral de lo que ocurre con las especies forestales amenazadas Níspero y Chanul, situación por lo demás notoriamente desfavorable a cualquier propósito de conservación de ambas especies, muy a pesar de la notoria mayor densidad poblacional del Níspero. Los datos muestran dos escenarios aparentemente contradictorios, por un lado se observa relativa baja densidad poblacional de las especies y por tanto la necesidad de emprender programas de conservación que garanticen su presencia en el largo plazo dentro de la zona, pero por otro lado, en comparación con lo que arrojaron otros estudios recientes sobre especies amenazadas en otros lugares del departamento del Chocó, se observa la relativa mejor situación de ambas especies en 
comparación con otras ya prácticamente agotadas en otros sitios otrora importantes desde el punto de vista forestal.

Estado de desarrollo. Lo concerniente al estado de desarrollo en el que se encuentran los individuos de las dos especies forestales amenazadas en Suruco Santa Mónica se presenta en la Tabla 4; de su lectura se desprenden dos hechos importantes, la excelente distribución de los individuos de la especie Níspero conforme los estados de desarrollo que presentan y la preocupante inexistencia de regeneración natural de la especie Chanul, que puede obedecer a problemas de tolerancia de la sombra que presentan las plántulas de esta especie.

Más del 50\% de la población de Níspero se encuentra en estado de brinzales lo que abre una luz de esperanza para el manejo de la especie, porque tal vez este se puede hacer sin tener que recurrir a la introducción de material vegetal de otros lugares, que podría presentar problemas de adaptación en futuros programas de repoblamiento con la especie. De igual manera, ilusiona el hecho de que 24 individuos de Níspero hayan pasado a estado de latizales sin que medie acción de manejo alguna para promocionarlos, lo cual puede ser indicativo de las necesidades de extremo cuidado únicamente en las primeras etapas del desarrollo de las plantas. Se debe procurar una revisión exhaustiva sobre los individuos en condición de fustales, pues al momento o incluso en el futuro cercano podrían ser proveedores de semillas para garantizar la permanencia de la especie en el lugar.
La no existencia de individuos de la especie Chanul en estado de desarrollo de brinzales y latizales es un claro signo de alarma que evidencia la necesidad de intervenir la especie en la vereda de Santa Mónica, pues la confinación de todos sus ejemplares a la condición de fustales pone a la especie en riesgo, dado que el aprovechamiento de los individuos existentes podría agotar la especie. Estos datos sugieren una veda inmediata al aprovechamiento de Chanul en el lugar, y en el caso de que se permisionara en sitios aledaños, debería realizarse una estricta vigilancia para que no fuera amparada la movilización de individuos aprovechados en lugares diferentes a los permitidos.

En lo que hace relación al estado de desarrollo de los ejemplares de especies forestales amenazadas en Puerto Salazar, Istmina, Chocó, se aprecia el bajo número de ejemplares de ambas especies en condición de brinzales y fustales, lo que evidencia problemas con la regeneración natural de estas especies. Aparentemente, los 42 individuos de la especie Níspero en estado de latizal podría tomarse como un caso excepcional a esta tendencia general, pero la existencia de sólo 12 brinzales impulsan la preocupación sobre su regeneración natural. La Tabla 5 muestra información detallada al respecto del tema.

De esta información integrada se destaca la especie Níspero, que presenta todavía en el área de estudio poblaciones de tamaño mediano; sin embargo, tal vez lo más importante es que los individuos tienen una distribución regular, la

Tabla 3

Presencia de las especies forestales amenazadas Níspero y Chanul en el Consejo Comunitario Mayor de Istmina, Chocó

\begin{tabular}{|c|c|c|c|c|}
\hline \multicolumn{2}{|c|}{ Nombre de la especie } & \multirow[t]{2}{*}{ Familia } & \multirow[t]{2}{*}{ Número de individuos } & \multirow{2}{*}{$\begin{array}{c}\mathbf{N}^{\circ} \text { de individuos por } \\
\text { hectárea }\end{array}$} \\
\hline común & científico & & & \\
\hline Níspero & Manilkara bidentata & Sapotaceae & 146 & 71.22 \\
\hline Chanul & Sacoglotis procera & Humiraceae & 33 & 16.10 \\
\hline Total & & & 179 & \\
\hline
\end{tabular}

Tabla 4

Estado de desarrollo de los individuos de especies forestales amenazadas en Suruco Santa Mónica, Istmina, Chocó

\begin{tabular}{lcccc}
\hline \multirow{2}{*}{ Especie } & \multicolumn{2}{c}{ Número } & \\
\cline { 2 - 4 } & brinzales & latizales & fustales & Total \\
\hline Chanul & 0 & 0 & 15 & 15 \\
Níspero & 48 & 24 & 11 & 83 \\
Total & & & & 98 \\
\hline
\end{tabular}


Bioetnia Volumen 8 No 2 (julio-diciembre), 2011

Tabla 5

Estado de desarrollo de los individuos de especies forestales amenazadas en Puerto Salazar (Chigorodó), Istmina, Chocó

\begin{tabular}{|c|c|c|c|c|}
\hline \multirow[t]{2}{*}{ Especie } & \multicolumn{3}{|c|}{ Número } & \multirow[b]{2}{*}{ Total } \\
\hline & brinzales & latizales & fustales & \\
\hline Chanul & 4 & 3 & 11 & 18 \\
\hline Níspero & 12 & 42 & 9 & 63 \\
\hline \multirow[t]{2}{*}{ Total } & & & \multicolumn{2}{|r|}{81} \\
\hline & \multicolumn{4}{|c|}{$\begin{array}{c}\text { Tabla } 6 \\
\text { Distribución de individuos por estado de desarrollo en el } \\
\text { Consejo Comunitario Mayor de Istmina, Chocó }\end{array}$} \\
\hline \multirow[t]{2}{*}{ Especie } & & Número & & \multirow[b]{2}{*}{ Total } \\
\hline & brinzales & latizales & fustales & \\
\hline Níspero & 86 & 37 & 23 & 146 \\
\hline Chanul & 4 & 4 & 25 & 33 \\
\hline Total & & & & 179 \\
\hline
\end{tabular}

Tabla 7

Distribución de individuos de Níspero y Chanul por clase diamétrica en la comunidad de Suruco Santa Mónica, municipio de Istmina, Chocó

\begin{tabular}{lccccccccc}
\hline \multirow{2}{*}{ Especie } & \multicolumn{8}{c}{ Clase diamétrica (cm) } & \multirow{2}{*}{ Total } \\
\cline { 2 - 7 } & $\mathbf{0 - 1 0}$ & $\mathbf{1 0 - 2 0}$ & $\mathbf{2 0 - 3 0}$ & $\mathbf{3 0 - 4 0}$ & $\mathbf{4 0 - 5 0}$ & $\mathbf{5 0 - 6 0}$ & $\mathbf{7 6 0}$ & \\
\hline Chanul & 0 & 4 & 2 & 3 & 5 & 1 & 0 & 15 \\
Níspero & 73 & 7 & 1 & 2 & 1 & 0 & 0 & 83 \\
Total & & & & & & & & 98 \\
\hline
\end{tabular}

mayoría se encuentran en estado de brinzales y latizales, situación que permite ver con esperanza el futuro de la especie, siempre que se haga un trabajo que le permita a los individuos ir avanzando hacia nuevos estadios de desarrollo que faciliten en el futuro la promoción del aprovechamiento de aquellos que presenten las mayores áreas basales.

El caso contrario lo constituye la especie Chanul, en la cual, el número total de individuos es relativamente bajo, con el agravante de que los brinzales se encuentran notablemente disminuidos y exclusivamente confinados al espacio geográfico de Puerto Salazar, sitio que en términos generales presenta menores posibilidades para la conservación de las especies forestales amenazadas, pero que claramente es más esperanzador si se trata de la conservación del Chanul. Aquí resulta evidente la necesidad de proteger los fustales, identificando los árboles semilleros y garantizando su permanencia para poderlos utilizar en futuros programas de manejo y fomento de la especie.
La Tabla 6 muestra el detalle de los datos obtenidos a partir del trabajo de campo para el estado de desarrollo de las especies forestales en condición de amenaza en el Consejo Comunitario Mayor de Istmina, Chocó.

Estructura diamétrica. En la Tabla 7 se puede apreciar la distribución de los individuos de las especies Níspero y Chanul por clase diamétrica en la vereda Santa Mónica del municipio Istmina, Chocó, territorio del Consejo Comunitario Mayor de Istmina, conocido como COCOMINSA.

La distribución de los individuos de especies amenazadas por clases diamétricas muestra de manera contundente la necesidad de proteger la especie Chanul, la cual, además de tener un escaso número de ejemplares, no tiene ninguno en la clase diamétrica inferior, situación que advierte sobre los riesgos en la renovación de la especie. De la misma forma, el hecho de que exista un alto porcentaje del total de los árboles de Chanul con diámetros de corta, obliga a su protección inmediata, a través de la sensibilización a los madereros y 
habitantes de la zona.

Los datos de la especie forestal Níspero, muestran una situación contraria a la encontrada para el Chanul, en tanto que casi el $90 \%$ de los individuos de la especie se encuentran en la clase diamétrica inferior, indicando que el futuro del Níspero en la zona depende mucho de la protección y el manejo que tengan estos ejemplares. Igualmente, la distribución de los individuos por clases diamétricas, obliga a mirar con atención la necesidad de proteger con mucho denuedo los tres ejemplares que presentan diámetros iguales o superiores a los 30 centímetros, pues en el futuro cercano, podrían ser los que produzcan el material vegetal para promover la conservación de la especie en la vereda.

En lo que respecta a la estructura diamétrica de las especies objeto de estudio en la vereda Puerto Salazar del Consejo Comunitario Mayor de Istmina, se destacan los siguientes hechos:

La especie Níspero tiene prácticamente el $100 \%$ de sus individuos en las dos primeras clases diamétricas, evidenciando también dificultades para alcanzar diámetros mayores; existen cuatro individuos entre los 30 y 60 centímetros de diámetro, los cuales se hace necesario proteger para evitar el agotamiento de la especie.

El análisis de la información parece indicar que en Puerto Salazar, Istmina, Chocó, se debe prohibir el aprovechamiento de las dos especies forestales amenazadas, más allá de las existencias que presentan y tomando más en cuenta la distribución de los individuos por clase diamétrica (Tabla 8).

La Tabla 9 muestra la distribución de los individuos de las especies forestales amenazadas por clase diamétrica. La especie Níspero presenta una distribución regular al presentar la mayor cantidad de individuos en las clases diamétricas inferiores, lo que indica la existencia de una buena cantidad de material vegetal de estas especies que tiene posibilidades de avanzar hacia estados más adultos en los que el volumen de madera sea deseable para emprender labores de aprovechamiento forestal. El Chanul presenta una distribución casi regular de individuos por clases diamétricas.

Orientaciones de manejo inmediato de las especies. Los datos que resultan del trabajo de campo conducen a la implementación inmediata de una veda al aprovechamiento de las dos especies forestales amenazadas (Níspero y Chanul), pues el estado de sus poblaciones en cuanto a densidad, condición de desarrollo y estructura diamétrica de los individuos así lo ameritan.

En el caso del Níspero la temporalidad de la veda puede ser menor, en tanto que hay ejemplares suficientes en los estadios más tempranos de desarrollo y en las clases diamétricas inferiores, lo que posibilitaría esperar el tiempo necesario para promocionar los 24 latizales a fustales, detectar cuál de los 11 fustales es proveedor de semillas, llevar los brinzales a latizales, y dirigir la germinación de semillas y el enriquecimiento del bosque con material vegetal traído de otros lugares cercanos.

En el caso del Chanul la veda ha de tener una mayor duración, porque tienen que mantenerse todos los individuos que se encuentran en condición de fustal para no acabar la especie en la vereda Santa Mónica, y hacer todo lo que sea

Tabla 8

Distribución de individuos de especies forestales amenazadas por clase diamétrica en Puerto Salazar (Chigorodó), Istmina, Chocó

\begin{tabular}{|c|c|c|c|c|c|c|c|c|}
\hline \multirow[t]{2}{*}{ Especie } & \multicolumn{7}{|c|}{ Clase diamétrica $(\mathrm{cm})$} & \multirow[t]{2}{*}{ Total } \\
\hline & 0-10 & $10-20$ & $20-30$ & $30-40$ & 40-50 & $50-60$ & $>60$ & \\
\hline Chanul & 7 & 5 & 1 & 2 & 1 & 2 & 0 & 18 \\
\hline Níspero & 57 & 2 & 0 & 2 & 1 & 1 & 0 & 63 \\
\hline \multicolumn{8}{|l|}{ Total } & 81 \\
\hline \multicolumn{9}{|c|}{$\begin{array}{c}\text { Tabla } 9 \\
\text { Distribución de las especies por clase diamétrica de las especies }\end{array}$} \\
\hline \multirow[t]{2}{*}{ Especie } & \multicolumn{7}{|c|}{ Clase diamétrica $(\mathrm{cm})$} & Total \\
\hline & 0-10 & $10-20$ & $20-30$ & $30-40$ & $40-50$ & $50-60$ & $>60$ & \\
\hline Níspero & 133 & 6 & 2 & 3 & 1 & 1 & & 146 \\
\hline Chanul & 10 & 7 & 5 & 3 & 7 & 1 & & 33 \\
\hline Total & & & & & & & & 179 \\
\hline
\end{tabular}




\section{Bioetnia Volumen 8 No 2 (julio-diciembre), 2011}

necesario para introducir y promocionar el crecimiento de plántulas que son las que en unos 20 años constituirán la masa forestal de Chanul en el lugar. También existe la posibilidad de adelantar una protección adicional especial de siete individuos que se encuentran en los estadios tempranos de desarrollo en la vereda Puerto Salazar.

\section{CONCLUSIONES}

La densidad poblacional y el estado de los individuos de las especies forestales Níspero (Manilkara bidenta) y Chanul (Sacoglotis procera) en el Consejo Comunitario Mayor de Istmina, evidencian signos de escasez, lo que obliga a pensar en orientaciones de manejo y decisiones de la autoridad ambiental departamental que promuevan su protección. A pesar de que los datos muestran un mejor estado de conservación del Níspero y el Chanul frente a otras especies forestales amenazadas con signos reales de agotamiento en el departamento del Chocó como son el Pino Amarillo, el Guayacán Amarillo y el Guayaquil, su situación amerita suficiente cuidado.

La situación particular del Níspero es mejor frente a lo que ocurre con el Chanul, un número de árboles más de cuatro veces mayor, una distribución regular de los individuos por clase diamétrica y una dominancia mayor del Chanul por tener mayor número de individuos en las clases diamétricas superiores demuestran la mayor vulnerabilidad de esta especie, razón por la cual se esperan diferencias así sean pequeñas, al momento de plantear las orientaciones de manejo.

De igual manera, se notan diferencias apreciables en el número de individuos por unidad de superficie para ambas especies entre las veredas Santa Mónica y Puerto Salazar, presentándose un mejor estado de conservación en la primera de ellas, en términos generales, pero a favor de la vereda Puerto Salazar en el caso particular del Chanul. Esta situación sugiere la declaratoria de vedas temporales diferenciales sobre su aprovechamiento, al tiempo que se promueven programas de manejo e investigaciones orientadas a garantizar la permanencia de estas especies.

\section{RECOMENDACIONES}

Se debe establecer un efectivo programa de monitoreo y control del aprovechamiento de especies restringidas, pues a pesar del grado de escasez y vulnerabilidad de las especies forestales, en algunos sitios de muestra se observaron actividades de aprovechamiento que van en contravía de cualquier medida de protección que pretenda llevarse a cabo.

El área donde se realizó el estudio podría ser objeto de aprovechamiento minero en el corto o mediano plazo, los niveles de intervención tan altos que presentan estas actividades productivas podrían dar al traste con las pocas existencias de las especies, lo que exige una inmediata intervención de la autoridad ambiental departamental; en este sentido, podría ser de mucha ayuda la georreferenciación y marcación de cada uno de los ejemplares que de las dos especies se encontraron.

Es imperante proteger y seleccionar los mejores árboles en la perspectiva de producción de material de propagación, lo que supone una etapa de trabajo subsiguiente. En los otros municipios del departamento deben emprenderse labores de investigación similares a las realizadas durante el desarrollo de este trabajo, con el fin de encontrar material que promueva la conservación de especies en inminente proceso de agotamiento, al igual que con el objeto de tomar decisiones por parte de la autoridad ambiental, que se orienten a conservar la base genética del departamento del Chocó.

\section{LITERATURA CITADA}

Andrade, M. G. 2001. Biodiversidad y conservación de la fauna colombiana. p. 35-47. En: Muñoz, P. (ed.). Memorias Primer Congreso Colombiano de Zoología. Bogotá: Instituto de Ciencias Naturales, Universidad Nacional de Colombia. 340 pp.

Calderón, E., G. Galeano, N. García (eds.). 2002. Libro Rojo de Plantas Fanerógamas de Colombia. Volumen 1: Chrysobalanaceae, Dichapetalaceae y Lecythidaceae. La serie Libros rojos de especies amenazadas de Colombia. Bogotá: Instituto Alexander von Humboldt, Instituto de Ciencias Naturales, Universidad Nacional de Colombia, Ministerio del Medio Ambiente.

Cárdenas, D. Diversidad florística en amazonia colombiana: Amenazas y acciones futuras. En: Colombia, 2010. IV Encuentro Nacional de Administración Ambiental: Biodiversidad en Colombia. Junio 5 de 2010, Bogotá.

Municipio de Istmina. 2008-2011. Esquema de Ordenamiento Territorial Municipio de Istmina. Municipio de Istmina. $256 \mathrm{pp}$.

Rivas, L. 2002. Historia del departamento del Chocó. Quibdó: Alcaldía Municipal de Quibdó. p. 81-8. 